\title{
Discovering Process Models through Relational Disjunctive Patterns Mining
}

\author{
Corrado Loglisci, Michelangelo Ceci, Annalisa Appice, Donato Malerba \\ Dipartimento di Informatica, Università degli Studi di Bari "Aldo Moro" \\ via Orabona, 4 - 70126 Bari - Italy \\ $\{$ loglisci,ceci,appice,malerba $\} @$ di.uniba.it
}

\begin{abstract}
The automatic discovery of process models can help to gain insight into various perspectives (e.g., control flow or data perspective) of the process executions traced in an event log. Frequent patterns mining offers a means to build human understandable representations of these process models. This paper describes the application of a multi-relational method of frequent pattern discovery into process mining. Multi-relational data mining is demanded for the variety of activities and actors involved in the process executions traced in an event log which leads to a relational (or structural) representation of the process executions. Peculiarity of this work is in the integration of disjunctive forms into relational patterns discovered from event logs. The introduction of disjunctive forms enables relational patterns to express frequent variants of process models. The effectiveness of using relational patterns with disjunctions to describe process models with variants is assessed on real logs of process executions.
\end{abstract}

Keywords-Process Mining; Disjunctive Patterns; Process Variants; Relational Data Mining; Frequent Pattern Discovery.

\section{INTRODUCTION}

Workflow management systems are becoming increasingly important in enterprises due to their capabilities of managing activities and actors involved in a business process as well as recording the logs of process executions.

Despite the amount of event logs produced by enterprises, software vendors use this information in order to answer to only simple questions under the assumption that the business process is fixed and known, e.g., the calculation of performance metrics like utilization and flow time. However, in many domains, business processes are evolving and people may have an oversimplified and incorrect view of the actual business processes [13]. In this scenario, process mining techniques play a key role with the extraction of models (or patterns) from event logs. These models can provide useful insights in the design of new workflows as well as they permit to collect information exploitable in the workflow optimization.

In the literature, several approaches for mining process models have been proposed. A Markovian approach is described in [3] to investigate the correspondence between the instances of a software engineering process and a model of the process. The process model is represented by a Finite State Machine derived from executions of software development processes and, with the help of distance metrics, it is used to quantitatively measure the discrepancies from new executions. A graph-based perspective is considered in [2] to analyze executions generated by workflow management systems and to generate process models as pure directed graphs expressing the precedence relationship between the activities of the processes. A particular kind of graphs, namely a variant of Petri nets called Workflow Nets, has been used in [14] to mine and model workflow processes. In these works, a sophisticated algorithm is presented to extract a process model based on binary relations discovered into the workflow logs. Notably, this approach permits to determine which class of workflow models the algorithm is guaranteed to work with. More recently, some authors [7], as we do in this work, have successfully applied pattern discovery techniques in order to identify frequent activities and their relationships. Since high frequency denotes regularity, frequent patterns can provide arguments for process models based on the evidence of regularities in the executions. Indeed, frequent patterns are intended as a means to capture the typical order of execution between activities (control perspective) and, at the same time, they model the possible associations among the properties of the process, activities and actors (data perspective).

The common characteristic of studies reported above is that they discover patterns (or more generally models) that identify the typical order of execution of activities without considering variants. However, real-world processes tend to be so complex and less structured that it is difficult to determine patterns to which several process executions comply with [15]. Moreover, the application of patterns that does not consider variants can turn out to be impractical in the workflow management systems which deal with exceptional situations and structural changes during runtime. On the other hand, considering these changes when discovering patterns may lead to the generation of numerous variants which are difficult to maintain even if slightly different one from each other [6], [9].

A deep analysis may reveal that this limitation comes from the fact that traditional frequent pattern discovery algorithms permit to mine conjunctions of the activities present in a set of process executions and does consider disjunctions that can model process variants. This approach poses some limitations to the patterns expressiveness and, in addition, leaves unexplored two potentialities of the pattern discovery: i) discovering interesting patterns when activities are not present in a sufficient number of process executions, and ii) discovering a combination of relationships between activities which are different from the classical conjunctions, such as 
disjunctions. These two potentialities are not independent each other, since the discovery of patterns including other relationships between activities may lead to discover patterns that otherwise would be discarded. Considering relationships among activities different from the classical conjunction would permit not only to consider variants in the process executions, but also to consider parallel executions of activities.

In this work, we extend our work in [1], where we have investigated the discovery of frequent patters as a means to extract a human interpretable representation of process models. The peculiarity of our previous work is that the frequent pattern discovery is performed in multi-relational data mining in order to take into account the intrinsic relational structure of logs: several activities and/or actors are involved in the same process execution. In particular, the multirelational approach permits to solve the following problems. First, objects collected in a log belong to different data types (executions, activities and actors) which interact one each other. By resorting to the first-order logic, that is one of the most common relational representation formalism, properties and interaction of executions, activities an actors are modeled by means of first-order logic predicates. Then, reasoning techniques developed in the field of inductive logic programming (ILP) are employed to discover patterns which are relationally defined as conjunctions of atomic formulas built using the data predicates. Second, activities stored in a log are marked with a timestamp which indicates the time of occurrence and implicitly defines a total temporal order over events. Temporal relationships between activities are represented as predicates and these predicates are used to investigate the temporal autocorrelation in the effect of a property of an activity/actor. Third, some user defined domain knowledge (e.g., the definition of the ordering relation between activities) may be available. This knowledge is profitably exploited by inferential mechanisms typical of a theorem prover which are integrated in the relational pattern discovery. However, the main disadvantage of the algorithm presented in [1] is that it cannot deal with process variants that, as stated before, are of fundamental importance in the problem at hand.

To cope with this further issue, we propose to derive process models through the discovery of relational patterns with disjunctions. The advantage of integrating disjunctive forms in patterns is two-fold. First, process variants can be identified and represented with the reference model, thus avoiding the explicit maintenance of numerous variants. Second, activities in the patterns can be $O R$-ed to represent typical $O R-$ split/OR - join in graph-based constructs [16].

The paper is organized as follows. In the next two sections we present related works and background. In Section IV we present our algorithm to discover disjunctive relational patterns. Experimental results on two real-world databases are commented in Section $\mathrm{V}$ and conclusions are drawn.

\section{RELATED WORKS}

Studies of frequent pattern discovery in Multi-Relational Data Mining are rooted in the field of ILP. In ILP both relational data and relational patterns are expressed in a first-order logic and the logical notions of generality order and of the downward/upward refinement operator on the space of patterns are used to define both the search space and the search strategy.

Recently, the multi-relational or ILP approaches to build business process models from event logs is receiving increasing attention. Goedertier et al. [5] have faced the task of predicting, by means of learned relational classification rules whether, given the state of a process instance, a particular state transition can occur. The representation formalism considered in this work is the Event Calculus, a first-order logic that elegantly captures the time-varying nature of facts. Learning is based on both positive information (possible transitions) and negative information (prohibited transitions). When no negative information is actually available in the logs, it is artificially generated by means of a sort of closed-world assumption (no pair of similar traces exists such that the transition of interest occurs). Similarly, Lamma et al. [8] have considered both compliant (positive information) and non compliant (negative information) execution traces and adapt the algorithm ICL [11] to learn constraints among activities (integrity constraints) expressed as logical formulas. In practice, the main problems of both methods are the reliable provision of negative information and their scalability to huge event logs. We overcome this issue by addressing a different task, that is, the frequent pattern discovery, where learning is from positive examples only.

Mining variants of a process is not a novel task. Indeed, significant studies have already addressed this task in the literature. Li et al. [9] describe an approach to discover the reference block-structured model which is the best in covering process variants. The model is obtained by first clustering activities in order to form blocks of similar activities, and then merging blocks into larger blocks. The final reference model is thus the model which has minimum distance from the variants, where the distance is measured by the number of change operations at the activity level. The brittleness of this work is the difficulty to create a set of only variants given that, in real event logs, traces of process and variants are stored together. The determination of a set of workflow models, called disjunctive workflow schema, is rather the solution proposed by Greco et al [6] to model the relevant variants. A stepwise procedure permits to refine models (workflow schema) created at the previous step, so that the final set is composed of hierarchically organized models. At each step, the executions which support a schema are partitioned into clusters each of which contains executions with the same characteristics. Each cluster thus represents a relevant variant and it is modeled by a refined workflow schema. However, since each variant is mined with a specialized model, the applicability of this method may be compromised in the case a huge number of specialized models is produced.

\section{BACKGROUND}

In [1], we have used SPADA in order to extract relational frequent patterns from event logs. In SPADA, it is possible to 
distinguish between reference objects ( $r o$ ) and task-relevant objects (tro). The former are data on which patterns are enumerated and contribute to compute the support of a pattern, while the latter contribute to define the former and they can be involved in a pattern. In the logic framework adopted by SPADA, event logs are converted into a deductive database $D$. Properties and relationships of the process executions (reference objects), activities and actors (task-relevant objects) are represented as ground atoms in the extensional part $D_{E}$, while a user defined background knowledge is expressed as a normal logic program which defines the intensional part $D_{I}$. An example of ground atoms stored into the extensional database $D_{E}$ is reported in the followings:

process $(e 1) \cdot \operatorname{process}(e 2)$.

activity $(e 1, a 1)$.activity $(e 1, a 2)$.

activity (e2,a3).activity $(e 2, a 4)$.

is a $(a 1$, workflow $)$ is a (a2, complete).

is a (a3, namemaker $)$.is a $(a 4$, schedule $)$.

time (a1, 10).time (a2, 25).time (a3, 22).time $(a 4,23)$.

actor (a1, paul).actor (a2, paul).is a (paul, user).

actor (a3, paul).actor (a4, mary).is a (mary, admin).

These ground atoms describe the process executions $e 1$ and $e 2$ (reference objects) according to the activities $a 1, a 2, a 3$, and $a 4$ (task-relevant objects) and the actors, $u 1$ and $u 2$ (taskrelevant objects). Differently, an example of a normal logic program stored as intensional database $D_{I}$ is the following:

$$
\begin{gathered}
\text { before }(A 1, A 2) \leftarrow \\
\operatorname{activity}(C, A 1), \operatorname{activity}(C, A 2), A 1 \neq A 2, \\
\operatorname{time}(A 1, T 1), \operatorname{time}(A 2, T 2), T 1<T 2, \\
\operatorname{not}(\operatorname{activity}(C, A), A \neq A 1, A \neq A 2, \\
\operatorname{time}(A, T), T 1<T, T<T 2)
\end{gathered}
$$

This normal logic program intensionally defines the temporal relationship before and permits to entail the temporal ground atoms before $(a 1, a 2)$ and before $(a 3, a 4)$ from $D_{E}$. Further examples of normal logic programs are the followings:

$$
\begin{aligned}
& \text { workflow }(A, B) \leftarrow \\
& \quad \operatorname{activity}(A, B), \text { is a }(B, \text { workflow }) \\
& \text { complete }(A, B) \leftarrow \\
& \quad \text { activity }(A, B), \text { is a }(B, \text { complete }) \\
& \ldots
\end{aligned}
$$

which permit to entail the ground atoms $\operatorname{workflow}(e 1, a 1)$, complete $(e 1, a 2)$.

The set of ground atoms (extensionally or intensionally) stored in $D$ is partitioned with respect to the process executions into a number of non-intersecting subsets $D[e]$ (units of analysis) each of which includes ground atoms concerning the activities and actors involved in the process execution $e$. Then, SPADA is able to discover relational frequent patterns across the units of analysis of $D$ which are associated to the process executions. The discovery process is in charge of a levelwise method, that is tailored as a breadth-first search in the lattice of relational patterns spanned by the $\theta$-subsumption generality order $\left(\succ_{\theta}\right)$. In this context, the relational patterns are formulated as follows:

$$
\operatorname{process}(P), \mu(P) \quad[s],
$$

where $P$ is a variable to represent a process execution, process $(P)$ is the atom that identifies a process execution $P$, while $\mu(P)$ is a conjunction of atoms which provides a description of a fragment of the process model underlying the generation of the process execution $P, s$ is the support of $P$ in $D$. Each atom in $\mu(P)$ represents one of the property of activity, actor or process execution, or relationship between activities, process executions and activities, activities and actors. For example:

$$
\begin{aligned}
& \operatorname{process}(P), \\
& \text { complete }(P, A), \text { schedule }(P, B), \text { delete }(P, C) \\
& \text { before }(A, B), \text { before }(B, C) . \quad[s=63 \%]
\end{aligned}
$$

is a relational pattern which describes a fragment of a process model where the activities complete, schedule and delete are executed in a sequence. The support $s$ estimates the probability $p($ process $(P) \cup \mu(P))$ on $D$. This means that $s \%$ of the units of analysis $D[e]$ are covered by process $(P) \cup \mu(P)$. Formally, the unit of analysis $D[e]$ is covered by $\operatorname{process}(P) \cup \mu(P)$ if there exists a substitution $\theta=\{P \leftarrow e\} \cdot \theta_{1}$ such that $[\operatorname{process}(P) \cup \mu(P)] \theta \subseteq D[e]$.

\section{Discovering Process Models with VARiants}

The motivation behind the usage of disjunctive forms in patterns is that the set of patterns discovered with traditional approaches, included SPADA, strongly depends on frequencybased thresholds such as the minimum support threshold. This means that when the minimum support is high valued, many interesting patterns are filtered out: conjunctions of atoms, for which the considered statistical measure does not exceed the minimum threshold, are ignored. The introduction of the disjunctive forms would permit to include the atoms which occur in parallel to or in alternative to other atoms. The effect is that of increasing the values of the statistical measures associated to the patterns. For example, let us suppose that the atom complete $(A, D)$ may occur alternatively to the atom schedule $(A, D)$. Then, the relational pattern:

$\operatorname{process}(A)$,

complete $(A, B),\langle\operatorname{complete}(\mathbf{A}, \mathbf{D}) \vee \operatorname{schedule}(\mathbf{A}, \mathbf{D})\rangle$,

before $(B, D)$

might be frequent, although the patterns:

process $(A)$, complete $(A, B)$, complete $(A, D)$, before $(B, D)$

$\operatorname{process}(A)$, complete $(A, B)$, schedule $(A, D)$, before $(B, D)$

might be both infrequent.

This consideration advocates the starting point of our approach, which is that of considering infrequent conjunctive patterns. These patterns are re-evaluated and extended to the disjunctive form by inserting disjunctions which involve atoms already present in the patterns. Disjunctions are created among atoms which are semantically related in the application domain. The semantic relatedness is intended as background knowledge on the predicates used in the atoms and permits us to numerically quantify the dissimilarity or conceptual distance between atoms. It guarantees that meaningful disjunctions are created.

The proposed approach follows a two-stepped procedure. First, it extracts the infrequent conjunctive patterns which 
can be considered as basis for the disjunctive patterns construction. In particular, patterns whose support is lower than the minimum support threshold, but exceeds a new ad-hoc threshold are selected. The newly defined threshold permits to identify the set of patterns to be extended to the disjunctive form. Second, by following the main intuition reported in [12], background knowledge is accommodated to exploit the information on the dissimilarity among the atoms in the disjunctive pattern generation. This way, disjunctive patterns are computed by iteratively integrating disjunctions into the patterns by means of a pair-wise joining operation. The final result is a set of patterns which may comprise both conjunctions and disjunctions of atoms, whose support is greater than the minimum support threshold.

Working in the relational setting adds additional sources of complexity to the problem of joining patterns due to the linkedness property [10]. In the relational representation, atoms of the same pattern are dependent each other due to the presence of variables (differently from the items in the propositional representation [12]). In this work, patterns to be joined should differ in only one atom (but different atoms should be similar) and share the remaining atoms up to a redenomination of variables. For example, let us consider the relational patterns:

$P 1: \operatorname{process}(A), \operatorname{complete}(A, B), \operatorname{complete}(A, D)$, before $(B, D)$. $P 2$ : $\operatorname{process}(A), \operatorname{complete}(A, B)$, schedule $(A, E)$, before $(B, E)$. The relational pattern:

$P 3: \operatorname{process}(A), \operatorname{complete}(A, B)$,

$\langle$ complete $(\mathbf{A}, \mathbf{W}) \vee \operatorname{schedule}(\mathbf{A}, \mathbf{W})\rangle$,

before $(B, W)$.

can be extracted according to the variable redenomination $D / W$ in $P 1$ and $E / W$ in $\mathrm{P} 2$.

Before formally defining the structure of the disjunctive relational patterns we consider in this work, we clarify how the deductive database we have described in the previous section changes. In particular, the intensional part $D_{I}$ of the deductive database $D$ includes the definition of a new kind of domain knowledge that permits to express the dissimilarity among atoms in the form of Datalog weighted edges of a graph. An example of the Datalog weighted edge is the following:

$$
\text { schedule - (complete - 0.88) }
$$

It states that the dissimilarity between the predicates schedule $(\cdot, \cdot)$ and complete $(\cdot, \cdot)$ is 0.88 . More generally, it represents an undirected edge $e$ between two vertices $v_{i}$ and $v_{j}$ (e.g., schedule, complete) with weight $w_{i j}$ (e.g., 0.88) and it is denoted as $e\left(v_{i}, v_{j}, w\right)$. A finite sequence of undirected edges $e_{1}, e_{2}, \ldots, e_{m}$ which links the vertices $v_{i}$ and $v_{j}$ is called path and denoted as $\rho\left(v_{i}, v_{j}\right)$. The complete list of such undirected edges represents the background information on the dissimilarity among atoms and allows the algorithm to join patterns by introducing disjunctions (e.g., $\langle\operatorname{schedule}(A, W) \vee$ complete $(A, W)\rangle)$.

The definition of a general-purpose dissimilarity measure between atoms is out of the scope of this paper. In this work, a domain expert has assigned a dissimilarity value to each pair of activities and each pair of actors.

A formal definition of the disjunctive relational pattern we consider in this work is reported in the following by using the set notation. In order to give this definition, some predicate symbols are introduced. They permit to express both properties and relationships of ro and tro and can be categorized into three classes: 1) key predicate identifies the ro in $D_{E}$ (i.e., the process, process $\left.(\cdot)\right)$; 2) property predicates are binary predicates which define the values taken by an attribute of a ro or of a tro;3) structural predicates are binary predicates which relate tro as well as ro with others tro (e.g., the process with the scheduling activity, schedule $(\cdot, \cdot)$ ).

Definition 4.1: A disjunctive relational pattern $P$ is a set of both atoms and disjunctions of atoms:

$$
\begin{aligned}
& \left\{p_{0}\left(t_{0}^{1}\right),\right. \\
& \left\langle p_{1}\left(t_{1}^{1}, t_{1}^{2}\right)\left|p_{2}\left(t_{2}^{1}, t_{2}^{2}\right)\right| \ldots\right\rangle, \\
& \ldots \\
& \left.\left\langle p_{k}\left(t_{k}^{1}, t_{k}^{2}\right)|\ldots| p_{k+h}\left(t_{k+h}^{1}, t_{k+h}^{2}\right)\right\rangle\right\}
\end{aligned}
$$

where $p_{0}$ is the key predicate, while $p_{i}, i=1, \ldots, k+h$, is either a structural predicate or a property predicate. The symbol " |" denotes the disjunctive operator " $\bigvee "$ ".

Terms $t_{i}^{j}$ are either constants, which correspond to values of property predicates, or variables, which identify either a ro or a tro. Each $p_{i}$ is a predicate occurring in $D_{E}$ (extensionally defined predicate) or defined in $D_{I}$ (intensionally defined predicate). An example of a disjunctive relational pattern expressed in the set notation is in the followings:

$P:\{$

$\operatorname{process}(A)$,

$\langle$ complete $(A, B)\rangle$,

$\langle$ complete $(A, W)|$ schedule $(A, W)\rangle$,

$\langle$ before $(B, W)\rangle$

\} .

where the variable $A$ denotes a $r o$, and variables $B$ and $W$ denote two tros. All variables are implicitly existentially quantified.

We now can give a formal statement of the problem of discovering relational frequent patterns with disjunctions. This problem is articulated in two steps.

\section{Given:}

the extensional part $D_{E}$ of the deductive database $D$ and the normal logic programs stored into the intensional part $D_{I}$ of $D$, two thresholds minSup $\in[0 ; 1]$ and nSup $\in[0 ; 1]$, where the former represents a minimum support value, while the latter represents a maximum support value (nSup < minSup),

Find: the collection $I_{R}$ of the relational infrequent patterns whose support is between $n$ Sup and minSup.

\section{Given:}

the collection $I_{R}$, the Datalog weighted edges stored in the intensional part $D_{I}$ of the deductive database $D$ and two thresholds minSup and $\gamma \in[0 ; 1](\gamma$ defines the maximum 
dissimilarity value among atoms involved into a disjunction), Find: relational patterns with disjunctions whose support exceeds minSup and whose dissimilarity of atoms involved in the disjunctions does not exceed $\gamma$.

The computational solution to these two sub-problems is implemented in jSPADA and presented in the following subsections.

\section{A. Mining Infrequent Conjunctive Relational Patterns}

In jSPADA, the search is based on the level-wise method and implements a two-stepped procedure: i) generation of candidate patterns with $k$ atoms ( $k$-th level) by considering the frequent patterns with $k-1$ atoms ( $k-1$-th level); ii) evaluation of the support of patterns with $k$ atoms. So, the patterns whose support does not exceeds minSup will be not considered for the next level: the patterns discarded (infrequent) at each level are rather considered for the generation of disjunctions. The collection $I_{R}$ is thus composed of a subset of infrequent patterns, more precisely those with support greater than or equal to $n S u p$ (and less than minSup).

We observe that, the set of infrequent patterns $I_{R}$ computed by jSPADA do not contain all possible infrequent relational patterns. This depends on the fact that any $k$-level infrequent pattern " $P_{k-1} \wedge A$ " output by jSPADA has the head $P_{k-1}$ that is a relational pattern frequent at the level $k-1$, and the tail $A$ which is an atom such that the conjunction " $P_{k-1} \wedge A$ " is infrequent. This means that infrequent patterns whose head is already infrequent cannot be discovered by jSPADA. On the other hand computing all possible infrequent relational patterns is computationally expensive. As a consequence, the set of patterns with disjunctions discovered from $I_{R}$ is a necessary approximation of the complete set of relational patterns with disjunctions.

\section{B. Extending Relational Patterns with Disjunctions}

The generation of disjunctive relational patterns is performed by creating disjunctions among similar atoms in accordance to the weighted edges of the background knowledge: two patterns which present similar atoms are joined to form only one. The implemented algorithm (see Algorithm 1) is composed of two sub-procedures: the first one (lines 2-12) creates a graph $\mathcal{G}_{\mathcal{D}}$ with the patterns of $I_{R}$ by exploiting the knowledge defined in $D_{I}$, while the second one (lines 13-32) joins two patterns (vertices) on the basis of the information (weight) associated to the connecting edge.

In particular, for each pair of patterns which have the same length (namely, at the same level of the level-wise search method) it checks whether they differ in only one atom and share the remaining atoms up to a redenomination of variables (line 3). Let $\alpha$ and $\beta$ be the two atoms differentiating $\mathrm{P}$ from $\mathrm{Q}(\alpha$ in $\mathrm{P}, \beta$ in $\mathrm{Q}$ ), a path $\rho$ which links $\alpha$ to $\beta$ (or vice-versa) is searched among the weighted edges according to $D_{I}$ : in the case the sum $\omega$ of the weights found in the path is lower than the maximum dissimilarity $\gamma$ the vertices $\mathrm{P}$ and $\mathrm{Q}$ are inserted into $\mathcal{G}_{\mathcal{D}}$ and linked through an edge with weight $\omega$ (lines 4-9). Note that when there is more than one path between $\alpha$ and $\beta$, then the path with lowest weight is considered. Intuitively, at the end of the first sub-procedure, $\mathcal{G}_{\mathcal{D}}$ will contain, as vertices, the patterns which meet the condition at the line 3 , and it will contain, as edges, the weights associated to the path linking the atoms differentiating the patterns.

Once we have $\mathcal{G}_{\mathcal{D}}$, a list $\mathcal{L}_{\mathcal{D}}$ is populated with the vertices and edges of $\mathcal{G}_{\mathcal{D}}$ : an element of $\mathcal{L}_{\mathcal{D}}$ is a triple $\langle P, Q, \omega\rangle$ composed of a pair of vertices-patterns $(P, Q)$ with their relative weight. Elements in $\mathcal{L}_{\mathcal{D}}$ are ranked in ascending order with respect to the values of $\omega$ so that the pairs of patterns with lower dissimilarity will be joined for first. This guarantees that disjunctions with very similar atoms will be preferred to the others (line 13). For each element of $\mathcal{L}_{\mathcal{D}}$ whose weight $\omega$ is lower than $\gamma$ the two patterns $P$ and $Q$ are joined to generate a pattern $J$ composed by the conjunction of the same atoms in common to the two patterns $P$ and $Q$ and of the disjunction formed by the two different (but similar) atoms (lines 14-15). This joining procedure permits to have patterns with the same length of the original ones and which occur when at least one of the original patterns occurs. Therefore, if a pattern $J$ is obtained by joining $P$ and $Q$, it covers a set of units of analysis equal to the union of those of $P$ and $Q$ : the support of $J$ is determined as in line 16 and, generally, it is higher than the support of both $P$ and $Q$. In the case the support of $J$ exceeds minSup, then it can be considered statistically interesting and no further processing is necessary (lines 16-17). Otherwise, $J$ is again considered and inserted into $\mathcal{G}_{\mathcal{D}}$ as follows. The edges which linked another pattern $R$ of $\mathcal{G}_{\mathcal{D}}$ to $P$ and $Q$ are modified in order to keep the links from $R$ to $J$ : the weight of the edges between one pattern $R$ and $J$ will be set to the average value of the weights of all the edges which linked $R$ to $P$ and $Q$ (lines 19-27). The modified graph $\mathcal{G}_{\mathcal{D}}$ contains conjunctive patterns (those of $I_{R}$ ) and patterns with disjunctions (those produced by joining). Thus, $\mathcal{G}_{\mathcal{D}}$ is re-evaluated for further joins and the algorithm proceeds iteratively (line 29-30) until no additional disjunction can be performed (namely, when $\mathcal{L}_{\mathcal{D}}$ is empty or the weights $\omega$ are higher than $\gamma$ ). At each iteration, the patterns $P$ and $Q$ are removed from $\mathcal{G}_{\mathcal{D}}$ (line 32).

An explanatory example is illustrated in Figure 1. Let us consider the background knowledge $D_{I}$ on the dissimilarity among four atoms and the set $I_{R}$ containing four infrequent conjunctive relational patterns as illustrated in Figure 1a and $\gamma$ equal to 0.7 . The first sub-procedure of Algorithm 1 analyzes $P_{1}, P_{2}, P_{3}, P_{4}$ and discovers that they differ in only one atom, while the other atoms are in common (process $(A)$, unknown $(A, C)$, before $(B, C))$. Then, it creates the graph $\mathcal{G}_{\mathcal{D}}$ by collocating $P_{1}, P_{2}$ and $P_{3}$ in three different vertices and linking them through edges whose weights are taken from the paths $\rho$ in $D_{I} . P_{4}$ is not considered because the dissimilarity between start and resume in the graph is higher than $\gamma$ (row (1) in Figure 1b). The second sub-procedure starts by ordering the weights of the edges: the first disjunction is created by joining $P_{1}$ and $P_{3}$ given that the dissimilarity value is lower than $\gamma$ and the lowest (row (2) in Figure 1b). Next, the pattern so created and $P_{2}$ are checked for joining. Both have the same length and differ in only one 


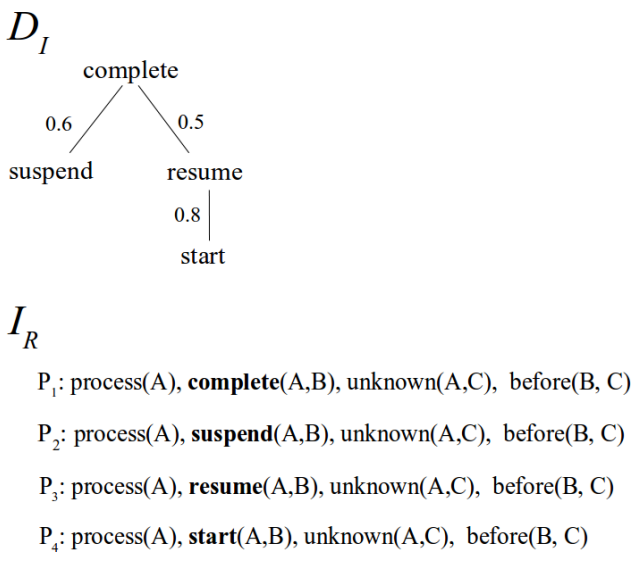

(a)

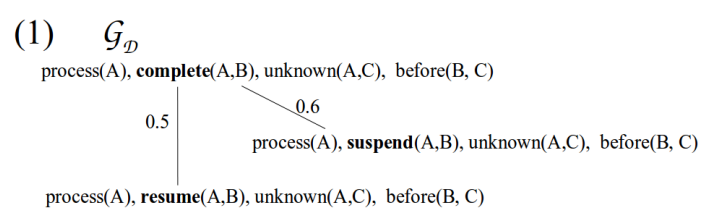

(2)

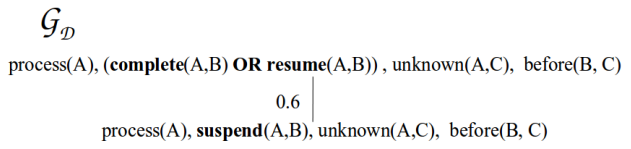

(3) $\mathcal{G}_{\mathcal{D}}$

process(A), (complete(A,B) OR resume(A,B) OR suspend(A,B)),unknown(A,C), before(B, C)

(b)

Fig. 1. An example of relational pattern extension by means of disjunctive atoms $(\gamma=0.7)$.

atom. Although the first presents a disjunction and the second presents a "simple" atom, dissimilarity is lower than $\gamma$ and a new disjunctive pattern is created (row (3) in Figure 1b).

A final consideration concerns the time complexity of Algorithm 1. Let us consider the set $I_{R}$ partitioned into disjoint subsets $\left\{I_{R k}\right\}_{k}$ on the basis of the pattern length $k$. Let $n_{k}$ be the cardinality of $I_{R k}$. For each $k$, the time complexity of extending relational patterns in $I_{R k}$ with disjunctions is quadratic in $n_{k}$ at worst (this is due to the pairwise join operation).

\section{EXPERIMENTS}

Experiments are performed by processing both the municipality event $\log$ publicly available on ProM web site ${ }^{1}$ and an event $\log$ provided by THINK3 Inc $^{2}$. The former concerns 374 executions of processes which handle the complaints (namely Afschriften) in a municipality in The Netherlands collected during the period May 4th 2005-November 8th 2005. The total number of activities is 9,174, while the number of distinct actors is 29. Activities are classified as complete (1,343), schedule (6,673), resume (178), start (809), suspend (166) and unknown (5). The latter describes 353,490 executions of business processes in a company. The period under analysis is from April 7th 2005 to January 10th 2007 for a total of 1,035,119 activities and 103 actors. Activities are classified as tools $(131)$, workflow $(919,052)$, namemaker $(106,839)$, delete $(2,767)$, deleteEnt $(2,354)$, prpDelete $(471)$, prpSmartDelete (53), prpModify (34) and cast $(1,430)$. Actors are classified as user (103), viewer (3) or administrator (2). In this Section, we illustrate the results obtained with a random sample of THINK3 data including 3580 executions. Process instances play the role of reference objects, while activities and actors play the role of task-relevant objects.

The goal of the experiments is to compare the conjunctive patterns discovered by SPADA with those disjunctive discovered by jSPADA in terms of the cardinality of the extracted

\footnotetext{
${ }^{1}$ http://is.tm.tue.nl/ cgunther/dev/prom/

${ }^{2}$ http://www.think3.com/en/default.aspx
}

patterns set and in terms of the learning time by varying threshold values. In the background knowledge $D_{I}$, a constant weight is assigned to each pair of activities. This way, the generation of a disjunctive form may equally involve each one of the activities. The weight is computed as the ratio of 1 (maximum dissimilarity value) to the number of distinct activities. In ProM, there are five distinct activities, then the weight is set to 0.2 (e.g., complete $\stackrel{0.2}{\leftrightarrow}$ resume). Similarly, in THINK3, the weight is set 0.16 for each one of the six activities, while it is set to 0.33 for each one of the three actors.

Experiments are performed ${ }^{3}$ by tuning the thresholds minSup, nSup and $\gamma$. Results are reported in Figure 2. As we can see in Figure 2.a $(n S u p=0.1 \%, \gamma=0.6)$ and Figure 2.d ( $n$ Sup $=30 \%, \gamma=0.7$ ), the number of final patterns (the summation of Conjunctive relational frequent patterns and Disjunctive relational frequent patterns) decreases as minSup increases. Indeed, by enlarging the range $[n S u p ; \min S u p)$, the number of infrequent conjunctive patterns and the number of potential disjunctive patterns grow up, while the increase of minSup leads to discover only those really frequent. An interesting consideration is inpired by the cardinality of sets of disjunctive frequent patterns obtained by varying minSup (Figure 2.a) and nSup (Figure 2.c): the order of magnitude of the number of discovered disjunctive patterns is constant and the size of the final set is reasonably small (lower than 10). This permits to reach the objective of discovering process models with variants which are not difficult to maintain and interpret for the end-user.

Another consideration can be found by the analysis of learning times (Figure 2.b) where it is possible to see that SPADA and jSPADA show comparable learning times. A deeper analysis reveals that by increasing minSup, the computational cost spent for the only generation of disjunctive patterns is of at least two orders of magnitude smaller than that of SPADA. Indeed, the increase of minSup leads to enlarge the range [nSup; $\operatorname{minSup}$ ) and this would require

\footnotetext{
${ }^{3}$ Data and results are accessible at http://www.di.uniba.it/ $\log$ lisci/jSPADA/.
} 


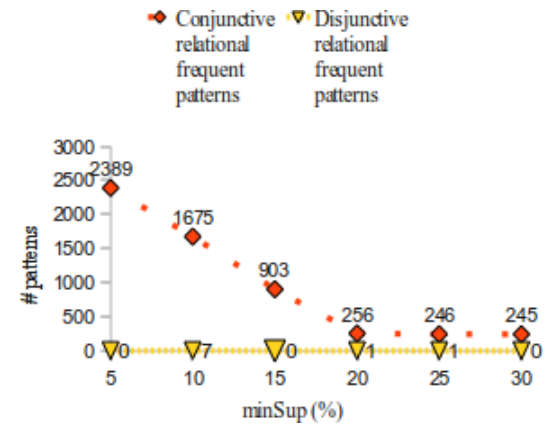

(a)

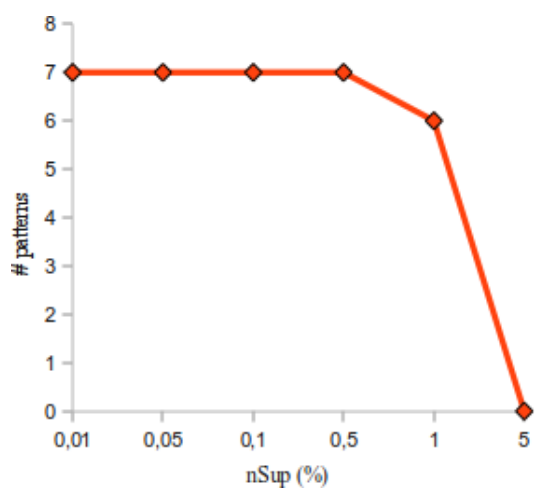

(c)

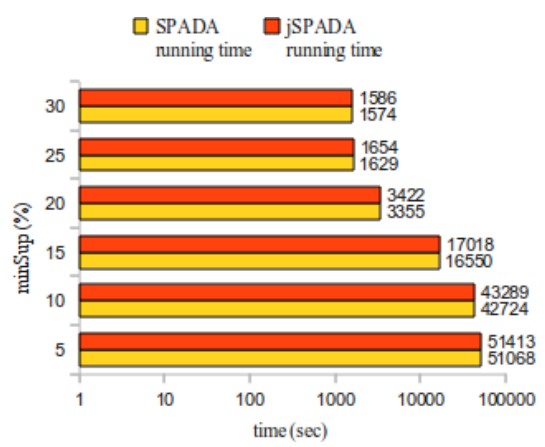

(b)

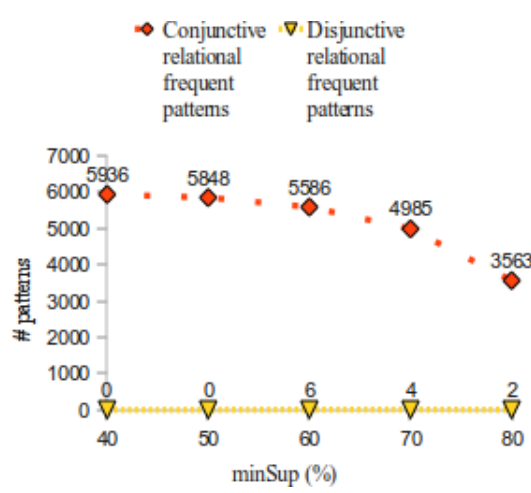

(d)

Fig. 2. Learning times and number of patterns discovered by SPADA and jSPADA by varying minSup and nSup for THINK3 (a,b,c) and ProM (d). Figures (a), (b) obtained with $n S u p=0.1 \%, \gamma=0.6$, Figure (c) obtained with $\min S u p=10 \%, \gamma=0.6$, Figure (d) obtained with $n S u p=30 \%, \gamma=0.7$.

longer learning time to process a greater set $I_{R}$. Actually, the reason of these time performances is twofold: first, a larger set $I_{R}$ does not necessarily imply a larger set of disjunctive patterns given that, if the atoms of two patterns are different, no disjunction can be created; second, when minSup has low values (e.g., 10\%) the number of iterations in Algorithm 1 is smaller since disjunctive patterns support easily exceeds minSup.

A peculiarity of the approach is that it enriches relational patterns discovered by SPADA with additional atoms. For instance, the following pattern is discovered by jSPADA at $\operatorname{minSup}=20 \%$ ( $n$ Sup $=0.001, \gamma=0.6$ in THINK3):

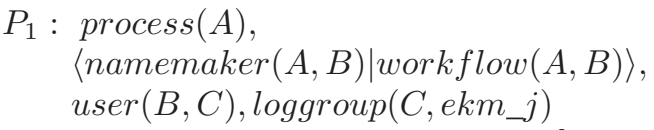

$$
[\text { support }=21.9 \%]
$$

$\mathrm{P}_{1}$ states that activities namemaker and workflow occur one in alternative to the other. This happens in 785 executions out of 3850 executions where the actor is of kind user and the login mode is $e k m \_j . \mathrm{P}_{1}$ joins the following two conjunctive patterns discovered by SPADA at $\operatorname{minSup}=5 \%$ that could represent two variants of the same model:

$P_{2}: \operatorname{process}(A)$, namemaker $(A, B)$, $\left.\operatorname{user}(B, C), \operatorname{loggroup}\left(C, e k m \_j\right){ }_{[\operatorname{support}}=8.1 \%\right]$
$P_{3}: \operatorname{process}(A)$, workflow $(A, B)$, $\operatorname{user}(B, C)$, loggroup $\left(C, e k m \_j\right)$ [support $=19.8 \%]$

Therefore, the proposed approach permits to unearth information extracted from SPADA at lower computational cost since the parameter minSup strongly affects the learning times of both systems. An example of more complex pattern is the following ( $\operatorname{minSup}=10 \%, n S u p=1 \%, \gamma=0.7$ in ProM):

$$
\begin{aligned}
P_{4}: & \operatorname{process}(A), \\
& \langle\operatorname{schedule}(A, D)| \text { complete }(A, D)\rangle \\
& \text { before }(D, C), \\
& \text { complete }(A, C), \\
& \quad \text { before }(C, B), \\
& \langle\operatorname{start}(A, B)| \text { schedule }(A, B))\rangle
\end{aligned}
$$$$
\text { [support }=12.1 \%]
$$

We observe that the disjunction (schedule or complete) on the activity $D$ is an example of $O R-$ split and $O R-j o i n$, while the disjunction (start or schedule) on the activity $B$ is an example of $O R-$ split. $\mathrm{P}_{4}$ has support $12.1 \%$ and expresses the order execution among three sets of activities: the first set, composed by two alternative or parallel activities schedule and complete, precedes a complete activity which precedes the third set composed by start and schedule.

\section{COnClusions}

In this paper, we present an approach to discover process models in the form of frequent relational patterns with dis- 


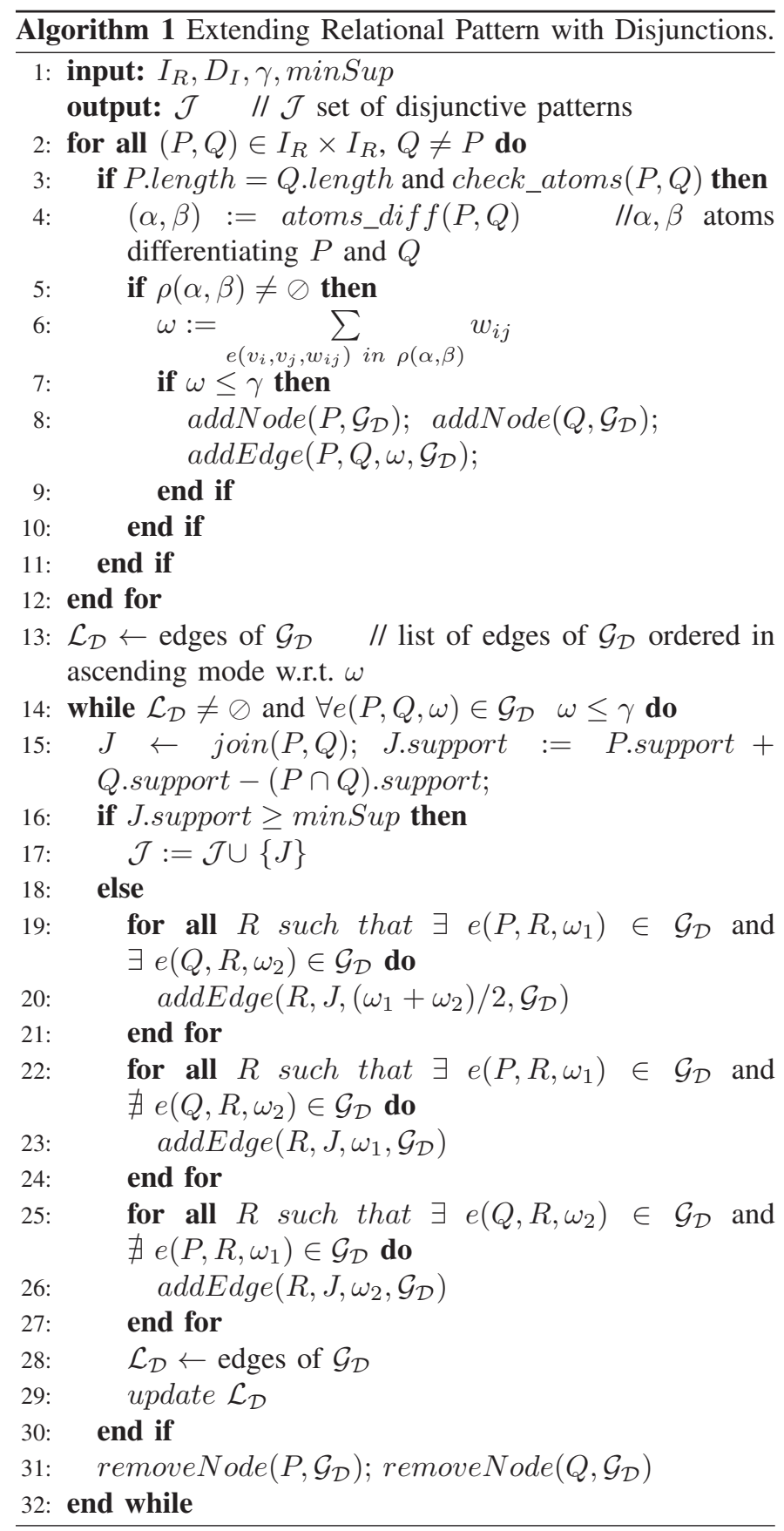

junctions. Patterns describe activities and actors involved in the process executions. The introduction of the disjunctive permits to express typical $O R-$ split/OR - join constructs such that variants of process models can be identified. It is noteworthy that relational patterns with disjunctive forms can improve the completeness [6] (the portion of executions in event log which are covered by a process model) of the conjunctive patterns. Indeed, disjunctive patterns are useful when executions are characterized by exceptions and changes.

Experiments on real event logs empirically prove the effectiveness of the proposed approach in efficiently discovering relational frequent patterns with disjunctions. Such patterns permit to express variants and can be easily maintained and interpreted by end-users. As future work, we intend to investigate the possibility of employing disjunctive patterns in the event log reduction. Additionally, we plan to derive a graphical model of the discovered patterns that might be more amenable for visualization.

\section{ACKNOWLEDGMENT}

This work is in partial fulfillment of the research objectives of the FAR project "DM19410: Laboratorio di Bioinformatica per la Biodiversità Molecolare" funded by the MIUR.

\section{REFERENCES}

[1] A. T. A. Appice, M. Ceci and D. Malerba. A parallel, distributed algorithm for relational frequent pattern discovery from very large data sets. Intelligent Data Analysis, 15(1):69-88, 2011.

[2] R. Agrawal, D. Gunopulos, and F. Leymann. Mining process models from workflow logs. In H.-J. Schek, F. Saltor, I. Ramos, and G. Alonso, editors, EDBT, volume 1377 of Lecture Notes in Computer Science, pages 469-483. Springer, 1998.

[3] J. E. Cook and A. L. Wolf. Software process validation: Quantitatively measuring the correspondence of a process to a model. ACM Trans. Softw. Eng. Methodol., 8(2):147-176, 1999.

[4] U. Dayal, J. Eder, J. Koehler, and H. A. Reijers, editors. Business Process Management, 7th International Conference, BPM 2009, Ulm, Germany, September 8-10, 2009. Proceedings, volume 5701 of Lecture Notes in Computer Science. Springer, 2009.

[5] S. Goedertier, D. Martens, B. Baesens, R. Haesen, and J. Vanthienen. A new approach for discovering business process models from event logs. In Technical Report. KBI 0716, 2007.

[6] G. Greco, A. Guzzo, L. Pontieri, and D. Sacca'. Discovering expressive process models by clustering log traces. IEEE Transactions on Knowledge and Data Engineering, 18:1010-1027, 2006.

[7] S.-Y. Hwang, C.-P. Wei, and W.-S. Yang. Discovery of temporal patterns from process instances. Comput. Ind., 53(3):345-364, 2004.

[8] E. Lamma, P. Mello, F. Riguzzi, and S. Storari. Applying inductive logic programming to process mining. In H. Blockeel, J. Ramon, J. W. Shavlik, and P. Tadepalli, editors, International Conference on Inductive Logic Programming,ILP 2007, volume 4894 of LNCS, pages 132-146. Springer-Verlag, 2007.

[9] C. Li, M. Reichert, and A. Wombacher. Discovering reference models by mining process variants using a heuristic approach. In Dayal et al. [4], pages 344-362.

[10] J. W. Lloyd. Foundations of Logic Programming, 2nd Edition. Springer, 1987.

[11] L. D. Raedt and W. V. Laer. Inductive constraint logic. In K. P. Jantke, T. Shinohara, and T. Zeugmann, editors, International Conference on Algorithmic Learning Theory, ALT 1995, volume 997 of LNCS, pages 80-94. Springer, 1995.

[12] J. F. Roddick and P. Fule. Semgram - integrating semantic graphs into association rule mining. In P. Christen and et al, editors, AusDM, volume 70 of CRPIT, pages 129-137. Australian Computer Society, 2007.

[13] W. van der Aalst, V. Rubin, H. Verbeek, B. van Dongen, E. Kindler, and C. Gnther. Process mining: a two-step approach to balance between underfitting and overfitting. Software and Systems Modeling, 9:87-111, 2010. 10.1007/s10270-008-0106-z.

[14] W. M. P. van der Aalst, H. A. Reijers, A. J. M. M. Weijters, B. F. van Dongen, A. K. A. de Medeiros, M. Song, and H. M. W. E. Verbeek. Business process mining: An industrial application. Inf. Syst., 32(5):713732, 2007.

[15] W. M. P. van der Aalst and A. J. M. M. Weijters. Process mining: a research agenda. Comput. Ind., 53(3):231-244, 2004.

[16] W. M. P. van der Aalst, T. Weijters, and L. Maruster. Workflow mining: Discovering process models from event logs. IEEE Trans. Knowl. Data Eng., 16(9):1128-1142, 2004. 